\title{
Expanding the SiMPl plasmid toolbox for use with spectinomycin/streptomycin
}

Navaneethan Palanisamy*, Jara Ballestin Ballestin, and Barbara Di Ventura*

Signalling Research Centres BIOSS and CIBSS, University of Freiburg, Schänzlestraße 18, 79104 Freiburg, Germany; Institute of Biology II, Faculty of Biology, University of Freiburg, Schänzlestraße 1, 79104 Freiburg, Germany.

*E-mail: navaneethan.palanisamy@biologie.uni-freiburg.de (NP) and barbara.diventura@biologie.uni-freiburg.de (BDV) 


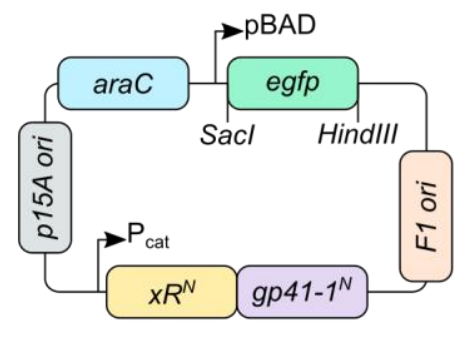

pSiMPI $^{\mathrm{X}} \mathrm{N}$

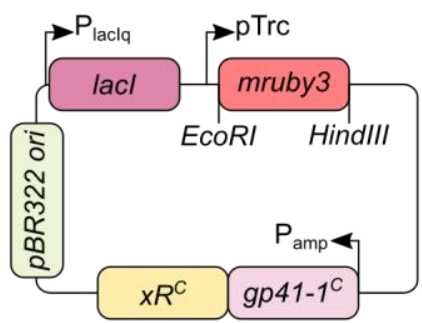

pSiMPI ${ }^{\mathrm{X}} \mathrm{C}$

Figure S1. Schematics of SiMPI plasmids. $\mathrm{pSiMPl}^{\mathrm{x}}{ }_{-} \mathrm{N}$ and $\mathrm{pSiMPl}^{\mathrm{x}}{ }_{-} \mathrm{C}$ are derived from pBAD33 and pTrc99a backbones, respectively. The chloramphenicol acetyl transferase gene of pBAD33 is replaced with $x R^{N}(\mathrm{~N}-$ terminal part of the split antibiotic resistance gene) followed by the sequence coding for the $\mathrm{N}$-terminal fragment of the split gp41-1 intein $\left(\mathrm{gp} 41-1^{\mathrm{N}}\right)$. The TEM-1 $\beta$-lactamase gene of $\mathrm{pTrc} 99 \mathrm{a}$ is replaced with the sequence coding for the C-terminal fragment of gp41-1 (gp41-1 ${ }^{\mathrm{C}}$ ) followed by $x R^{C}$ (the C-terminal part of the split antibiotic resistance gene). Restriction sites that can be used to swap the reporter genes with any gene of interest are shown. 


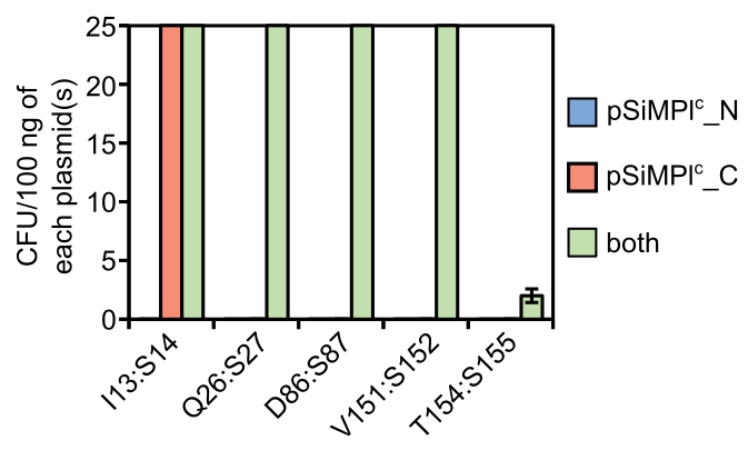

Figure S2. Chloramphenicol acetyltransferase split at T154:S155 is functional. Zoom in of the bar graph shown in Figure 1d. The transformation efficiency of the $\mathrm{pSiMPI}^{\mathrm{c}}$ plasmids in chemically competent $E$. coli TOP10 cells was assessed on chloramphenicol selective plates after Gibson Assembly. Values represent mean \pm S.E.M. of three independent experiments. 

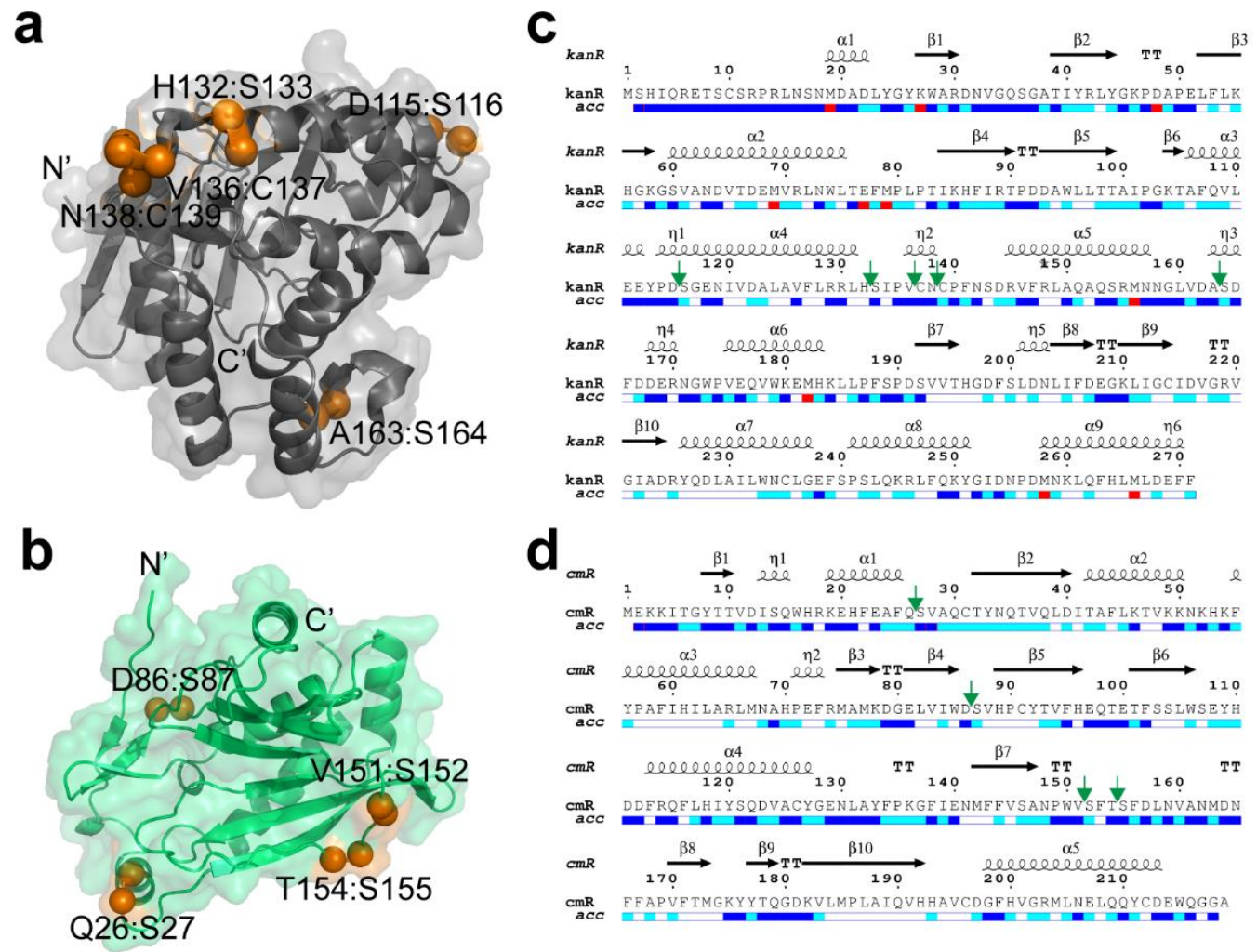

Figure S3. The identified splice sites in aminoglycoside $3^{\prime}$-phosphotransferase and chloramphenicol acetyltransferase are surface accessible. (a-b) Cartoon-surface representation of the crystal structure of aminoglycoside 3'-phosphotransferase (PDB ID: 4ej7; a) and chloramphenicol acetyltransferase (PDB ID: 1q23; b). The residues at the splice site are indicated by orange spheres. Structures were depicted in PyMOL (PyMOL Molecular Graphics System, v. 1.8.x, Schrödinger, LLC). (c-d) Relative accessibility (acc) of each residue in aminoglycoside 3'-phosphotransferase (c) and chloramphenicol acetyltransferase (d). Secondary structural elements are represented by black arrows and coils. The image was created using the ESPript 3.0 server $^{l}$. The color code is as follow: blue $=$ accessible, cyan $=$ partially accessible, white $=$ buried, red $=$ not predicted. Splice site are indicated by green arrows. 
a

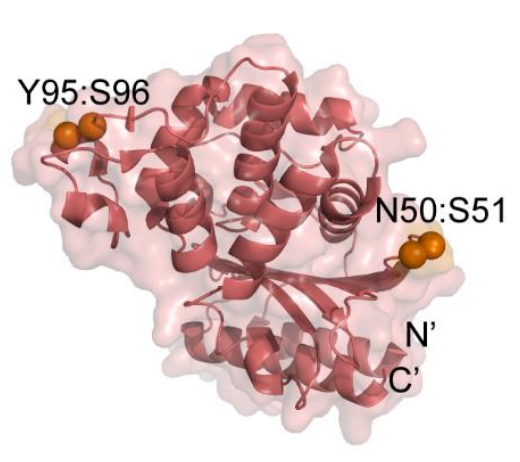

b

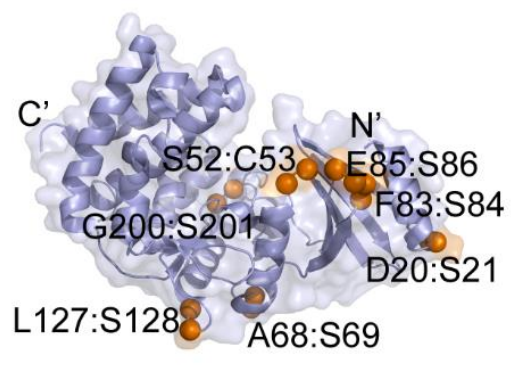

C

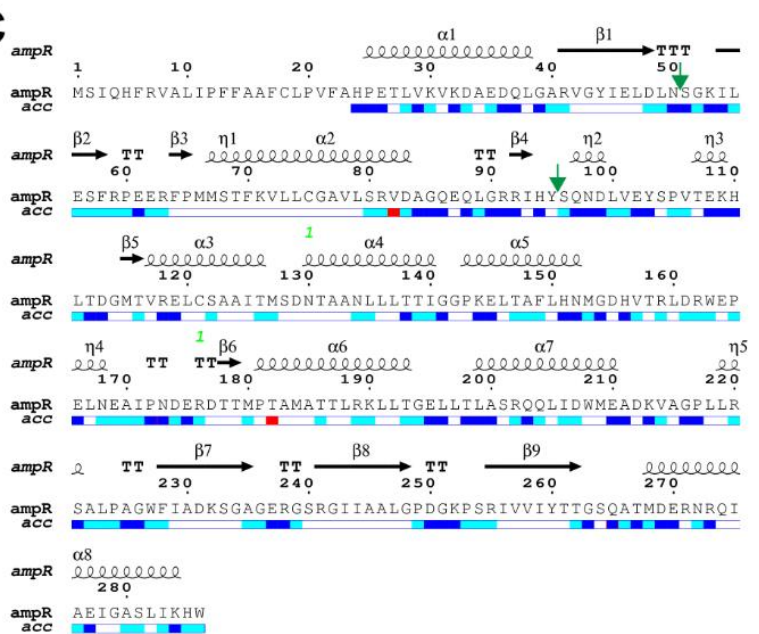

d

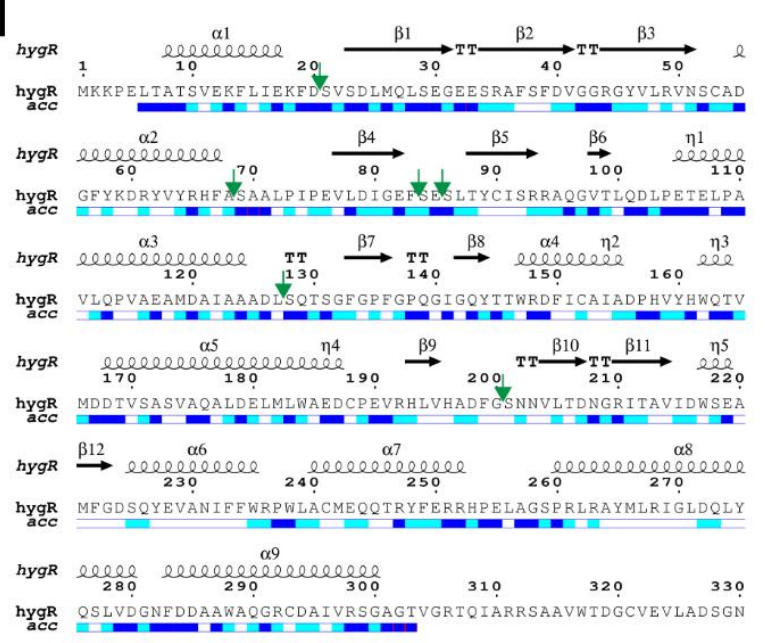

Figure S4. The identified splice sites in TEM-1 $\beta$-lactamase and hygromycin B phosphotransferase are surface accessible. (a-b) Cartoon-surface representation of the crystal structure of TEM-1 $\beta$-lactamase (PDB ID: 1zg4; a) and hygromycin B phosphotransferase (PDB ID: $3 \mathrm{w} 0 \mathrm{~s} ; \mathbf{b}$ ). The residues at the splice site are indicated by orange spheres. Structures were depicted in PyMOL (PyMOL Molecular Graphics System, v. 1.8.x, Schrödinger, LLC). (c-d) Relative accessibility (acc) of each residue in TEM-1 $\beta$-lactamase (c) and hygromycin B phosphotransferase (d). Secondary structural elements are represented by black arrows and coils. The image was created using the ESPript 3.0 server $^{l}$. The color code is as follow: blue = accessible, cyan = partially accessible, white $=$ buried, red $=$ not predicted. Splice site are indicated by green arrows . 

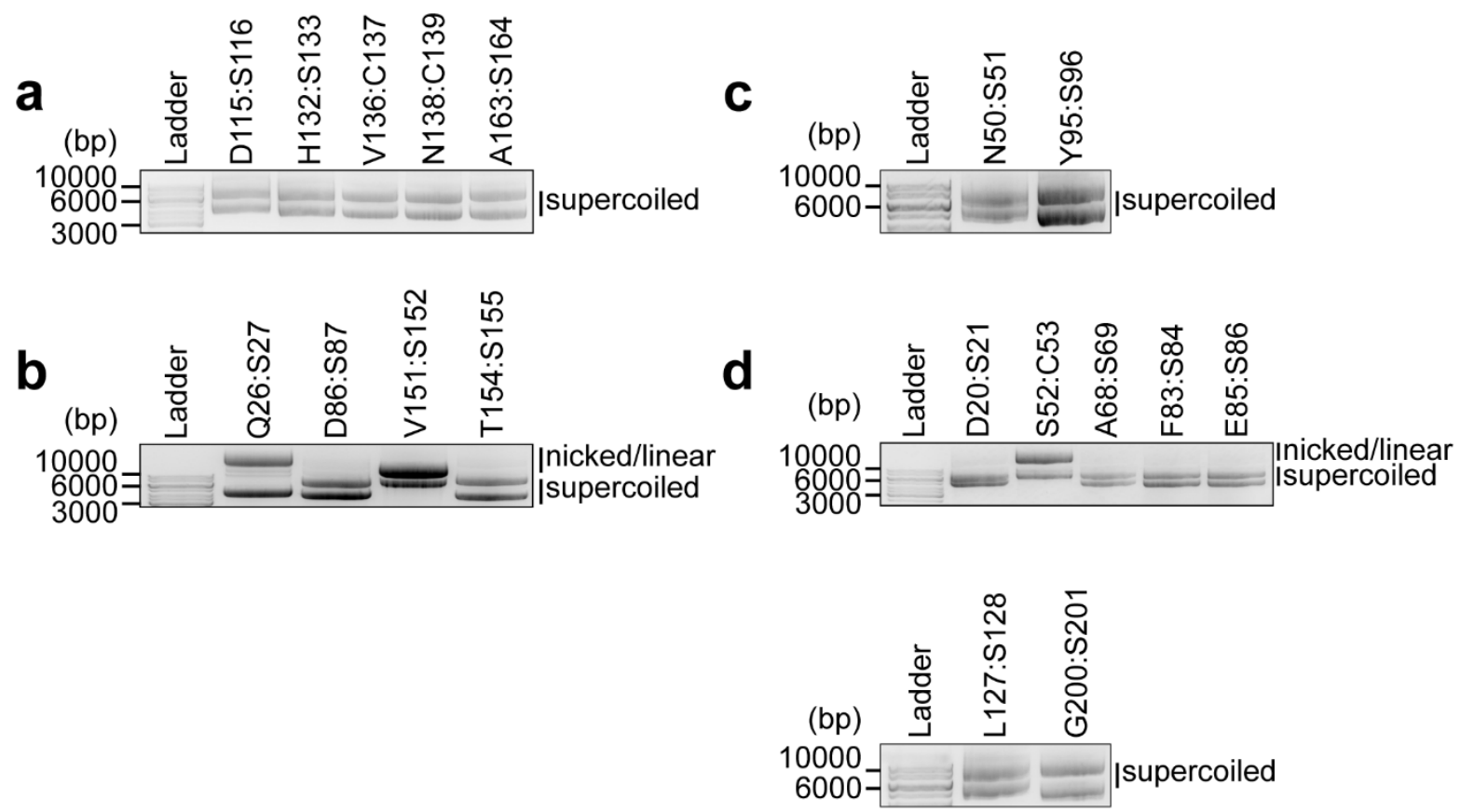

Figure S5. Both pSiMPI plasmids are present in the cells. (a-d) Representative ethidium bromide-stained $1 \%$ agarose gel showing that both plasmids are recovered after plasmid DNA extraction from a random bacterial clone. Cells were transformed with the pSiMPl plasmid pair for use with kanamycin (a), chloramphenicol (b), ampicillin (c) and hygromycin (d). 

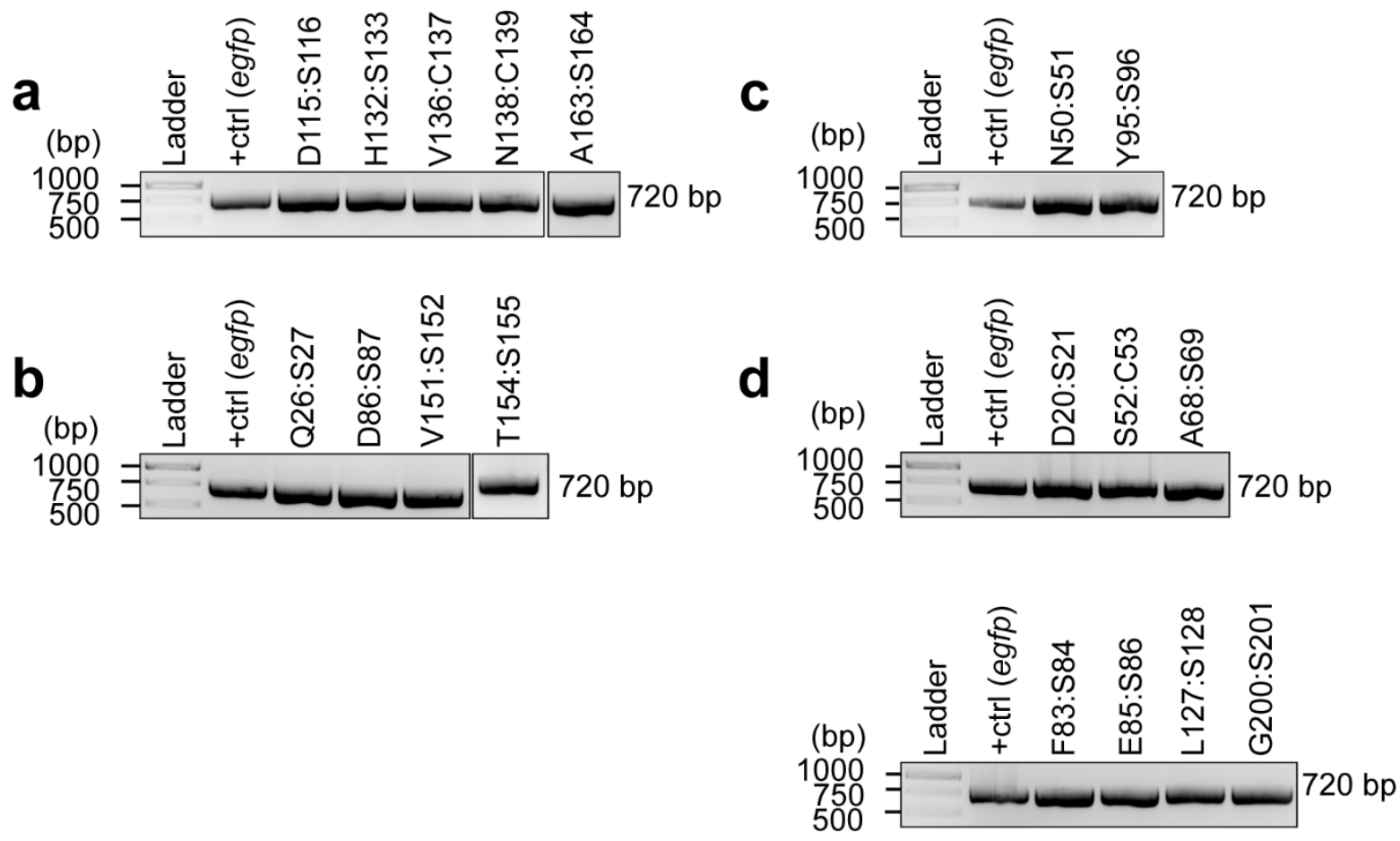

Figure S6. The egfp gene encoded on pSiMPI_N is present in the cells transformed with pSiMPI plasmid pairs. (a-d) Representative ethidium bromide-stained 1\% agarose gel showing the PCR product obtained with egfp-specific primers. The PCR was performed on the plasmid DNA extracted from a random clone of cells transformed with the pSiMPl pair for use with kanamycin (a), chloramphenicol (b), ampicillin (c) and hygromycin (d). egfp cloned into pBAD33 was used as positive control (+ ctrl). 

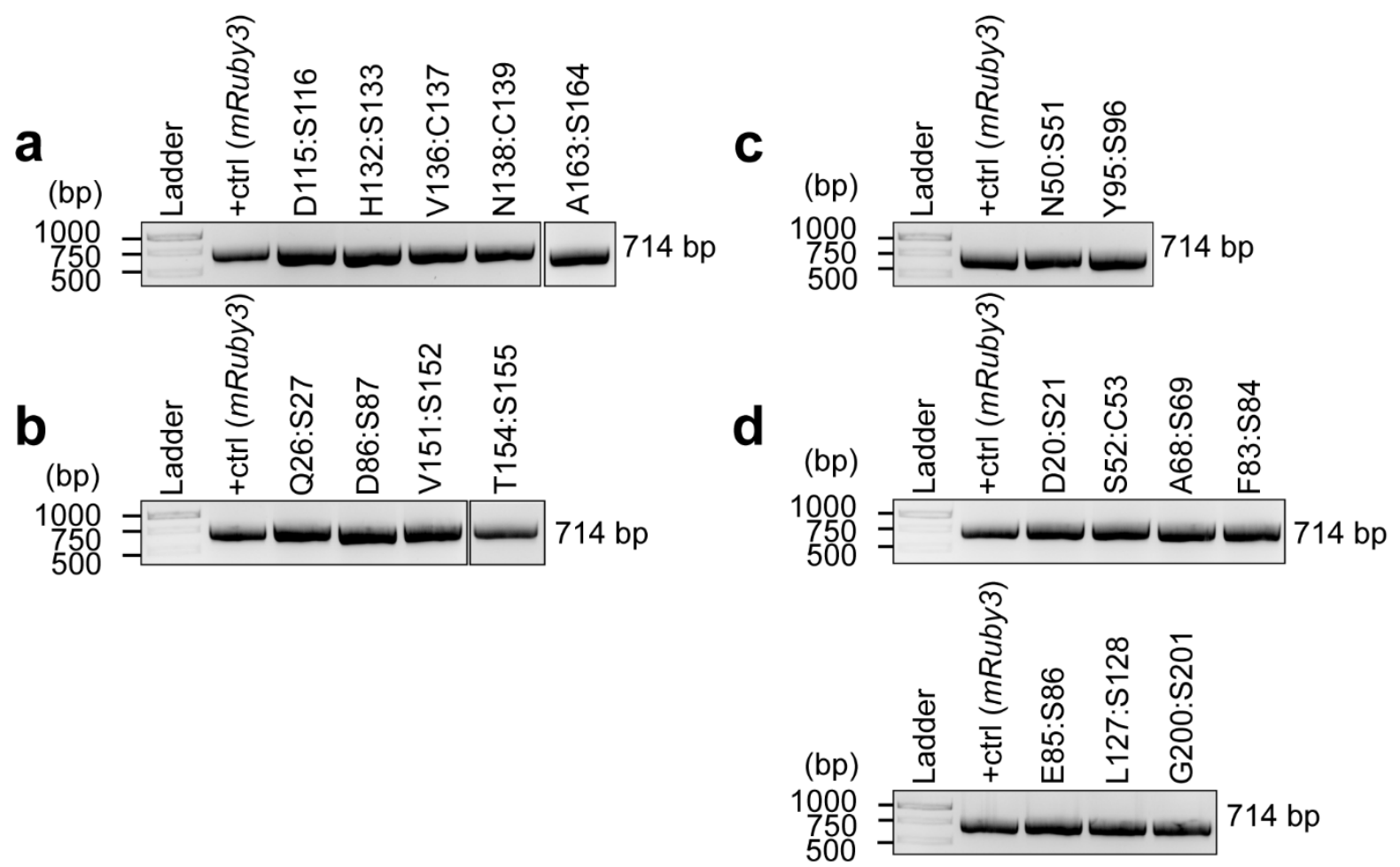

Figure S7. The mruby3 gene encoded on pSiMPI_C is present in the cells transformed with pSiMPI plasmid pairs. (a-d) Representative ethidium bromide-stained 1\% agarose gel showing the PCR product obtained with mruby3-specific primers. The PCR was performed on the plasmid DNA extracted from a random clone of cells transformed with the $\mathrm{pSiMPl}$ pair for use with kanamycin (a), chloramphenicol (b), ampicillin (c) and hygromycin (d). mruby3 cloned into pTrc99a was used as positive control (+ ctrl). 

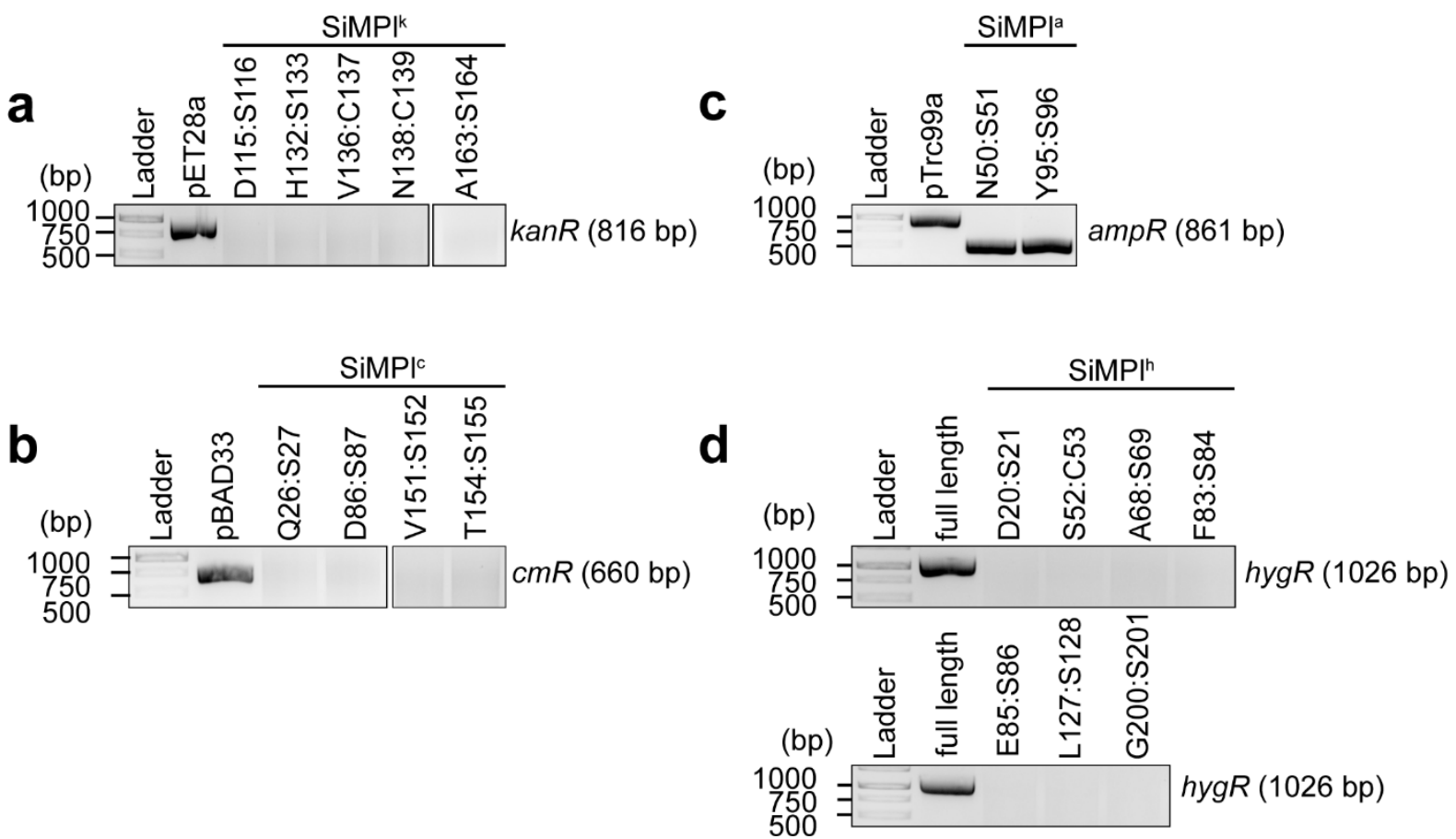

Figure S8. A full-length resistance marker gene is not found in cells transformed with pSiMPI plasmid pairs. (a-d) Representative ethidium bromide-stained 1\% agarose gel showing the product of a PCR amplification with primers specific for the $k a n R(\mathbf{a}), \operatorname{cm} R(\mathbf{b}), \operatorname{ampR}(\mathbf{c})$ and $h y g R(\mathbf{d})$ genes, respectively. The PCR was performed on plasmid DNA extracted from cells transformed with the pSiMPl plasmid pairs or single plasmids encoding the full-length resistance genes, which were used as positive controls (pET28a, $k a n R$; pBAD33, cmR; pTrc99a, ampR; pBAD33 into which the $c m R$ gene was swapped with the $h y g R$ one, hygR). (c) pBAD33 carries a defunct $a m p R$ gene. So, you also see amplicons albeit smaller than the full-length gene. 

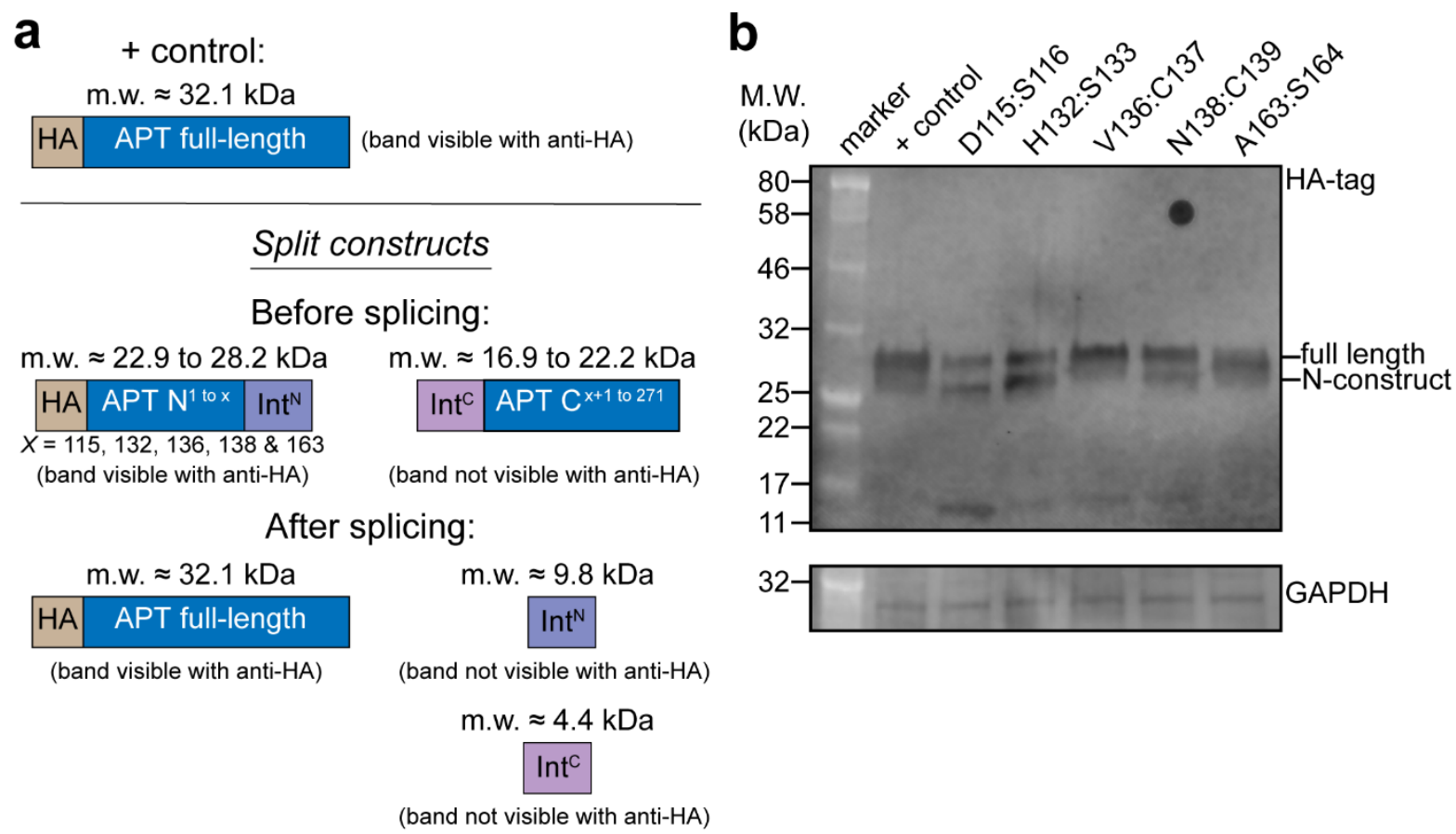

Figure S9. Aminoglycoside 3'-phosphotransferase (APT) is reconstituted by gp41-1-mediated protein splicing. (a) Schematics showing polypeptide fragments with predicted molecular weights. Note that APT did not tolerate a second tag at its C-terminus. This prevented us from detecting the C-terminal construct in the Western blot. The spliced product could be, however, detected. (b) Representative Western blot. The membrane was scanned with two different lasers to detect AlexaFluor 488-conjugated anti-rat secondary antibody directed against the rat anti-HA antibody (upper image) and AlexaFluor 790-conjugated anti-mouse secondary antibody directed against mouse anti-GAPDH (lower image). GAPDH was used as loading control. 

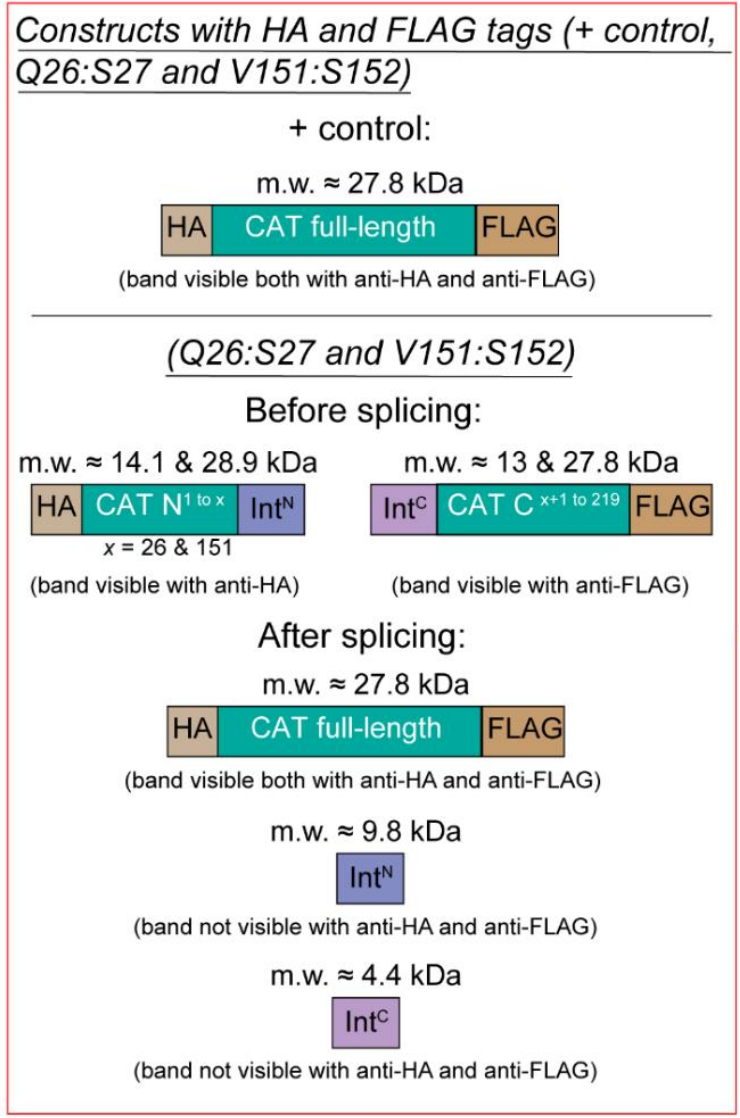

\section{Construct with only HA tag (D86:S87) \\ Before splicing:

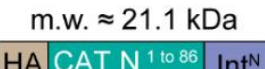 \\ (band visible with anti-HA)

\begin{tabular}{|c|c|}
\hline $\mathrm{m} . \mathrm{I}$ & I. $\approx 19.8 \mathrm{kDa}$ \\
\hline $\operatorname{lnt} t^{C}$ & CAT C 87 to 219 \\
\hline
\end{tabular} \\ (band not visible with anti-HA)}

After splicing:

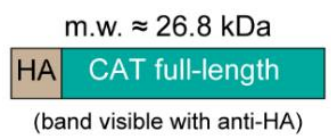

$$
\begin{gathered}
\text { m.w. } \approx 9.8 \mathrm{kDa} \\
\text { Int } \\
\text { (band not visible with anti-HA) } \\
\text { m.w. } \approx 4.4 \mathrm{kDa} \\
\text { Int }^{\mathrm{C}} \\
\text { (band not visible with anti-HA) }
\end{gathered}
$$

\section{Construct with only FLAG tag (T154:S155)}

Before splicing:

m.w. $\approx 28.1 \mathrm{kDa}$

\begin{tabular}{|l|l|l|l|l|}
\hline CAT $N^{1}$ to 154 & Int $^{\mathrm{N}} \quad \operatorname{lnt}^{\mathrm{C}}$ & CAT C & 155 to 219 & FLAG \\
\hline
\end{tabular}

(band not visible with anti-FLAG) (band visible with anti-FLAG)

\section{After splicing:}

$$
\text { m.w. } \approx 26.7 \mathrm{kDa} \quad \text { m.w. } \approx 9.8 \mathrm{kDa}
$$

\section{\begin{tabular}{l|l|l|l|l} 
CAT full-length & FLAG & $\operatorname{lnt}^{\mathrm{N}}$
\end{tabular}}

(band visible with anti-FLAG) (band not visible with anti-FLAG)

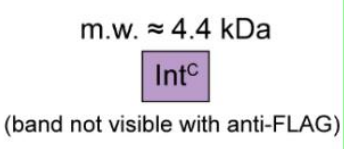

b
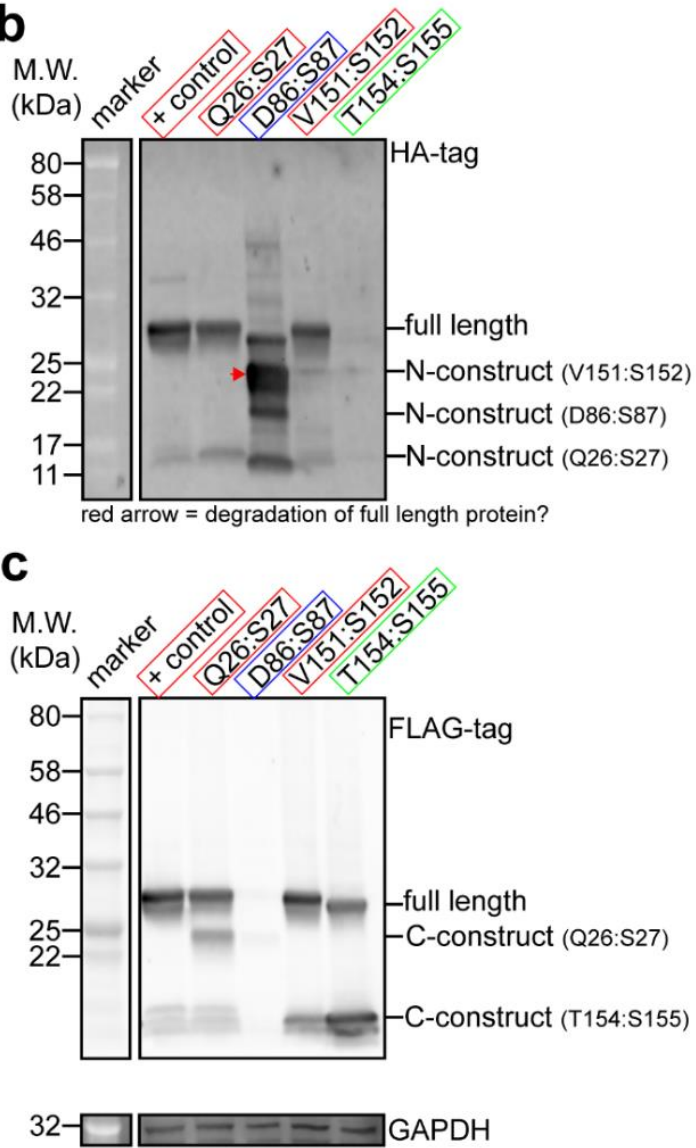

Figure S10. Chloramphenicol acetyltransferase (CAT) is reconstituted by gp41-1-mediated protein splicing. (a) Schematics showing polypeptide fragments with predicted molecular weights. Note that for some of the split constructs, we were not able to tag the enzyme at both termini (no clones were obtained after transformation). Those constructs tolerated either only the HA tag at the N-terminus (D86:S87; blue rectangle), or the FLAG tag at the C-terminus (T154:S155; green rectangle). (b,c) Representative Western blot. The membrane was scanned with three different lasers to detect AlexaFluor 488-conjugated anti-rat secondary antibody directed against the rat anti-HA antibody (upper image), Cyanine5-conjugated anti-rabbit secondary antibody directed against the rabbit anti-FLAG antibody (middle image), and AlexaFluor 790-conjugated antimouse secondary antibody directed against the mouse anti-GAPDH antibody (lower image). GAPDH was used as loading control. The red arrow indicates a potential degradation product for the construct split at D86:S87. 
a

+ control:

m.w. $\approx 30 \mathrm{kDa}$

TEM-1 $\beta$-L full-length FLAG

(band visible with anti-FLAG)

\section{Split constructs}

Before splicing:

\section{m.w. $\approx 12.8 \& 18 \mathrm{kDa}$

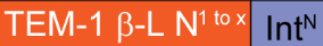

$$
X=50 \& 95
$$

(band not visible with anti-FLAG) m.w. $\approx 31.2 \& 26.1 \mathrm{kDa}$ \begin{tabular}{|l|l|l|}
\hline Int $^{\mathrm{C}}$ & TEM-1 $\beta-L \mathrm{C}^{\mathrm{x}+1}$ to 286 & FLAG \\
\hline
\end{tabular}

(band visible with anti-FLAG)

\section{b}

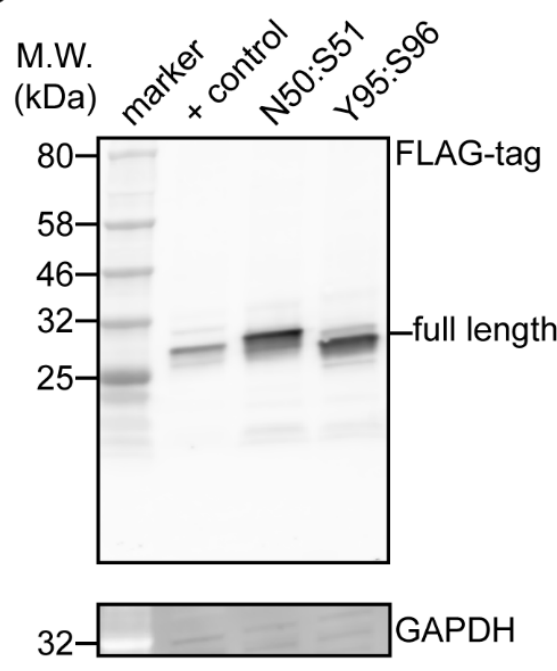

\section{TEM-1 $\beta$-L full-length FLAG}

(band visible with anti-FLAG)

\author{
m.w. $\approx 9.8 \mathrm{kDa}$ \\ $\operatorname{lnt}^{\mathrm{N}}$ \\ (band not visible with anti-FLAG) \\ m.w. $\approx 4.4 \mathrm{kDa}$ \\ Int $^{\mathrm{C}}$ \\ (band not visible with anti-FLAG)
}

Figure S11. TEM-1 $\beta$-lactamase (TEM-1 $\beta$-L) is reconstituted by gp41-1-mediated protein splicing. (a) Schematics showing polypeptide fragments with predicted molecular weights. Note that at the N-terminus of TEM-1 $\beta$-lactamase (TEM-1 $\beta$-L) a secretion tag that targets it to the periplasm is present; therefore, we were not able to include the $\mathrm{N}$-terminal HA tag in this construct. This prevented us from detecting the $\mathrm{N}$-terminal construct in the Western blot. The spliced product could be, however, detected. (b) Representative Western blot. The membrane was scanned with two different lasers to detect Cyanine5-conjugated anti-rabbit secondary antibody directed against the rabbit anti-FLAG antibody (upper image) and AlexaFluor 790-conjugated anti-mouse secondary antibody directed against mouse anti-GAPDH (lower image). GAPDH was used as loading control. Unfortunately, the antibody was not always reliable. Despite being the same batch, we experienced several problems with the detection (compare for instance Figures S10 and S11). 
a + control:

$\mathrm{m} . \mathrm{w} . \approx 38 \mathrm{kDa}$

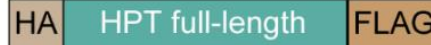

(band visible both with anti-HA and anti-FLAG)

\section{Split constructs \\ Before splicing:}

\begin{tabular}{|c|c|c|c|c|c|}
\hline \multicolumn{3}{|c|}{$\mathrm{m} . \mathrm{w} . \approx 13.3$ to $33 \mathrm{kDa}$} & \multicolumn{3}{|c|}{ m.w. $\approx 21.2$ to $41 \mathrm{kDa}$} \\
\hline HA & HPT $N^{1 \text { to } x}$ & $\operatorname{lnt} t^{N}$ & $\operatorname{lnt}^{\mathrm{C}}$ & HPT $\mathrm{C}^{\mathrm{x}+1}$ to 341 & FLAG \\
\hline
\end{tabular}

After splicing:

m.w. $\approx 38 \mathrm{kDa}$

HA HPT full-length FLAG

(band visible both with anti-HA and anti-FLAG)

m.w. $\approx 4.4 \mathrm{kDa}$

$\operatorname{lnt}^{\mathrm{C}} \begin{aligned} & \text { (band not visible with anti-HA and } \\ & \text { anti-FLAG) }\end{aligned}$

b $\operatorname{Int}^{\mathrm{N}} \begin{aligned} & \text { (band not visible with anti-HA and } \\ & \text { anti-FLAG) }\end{aligned}$

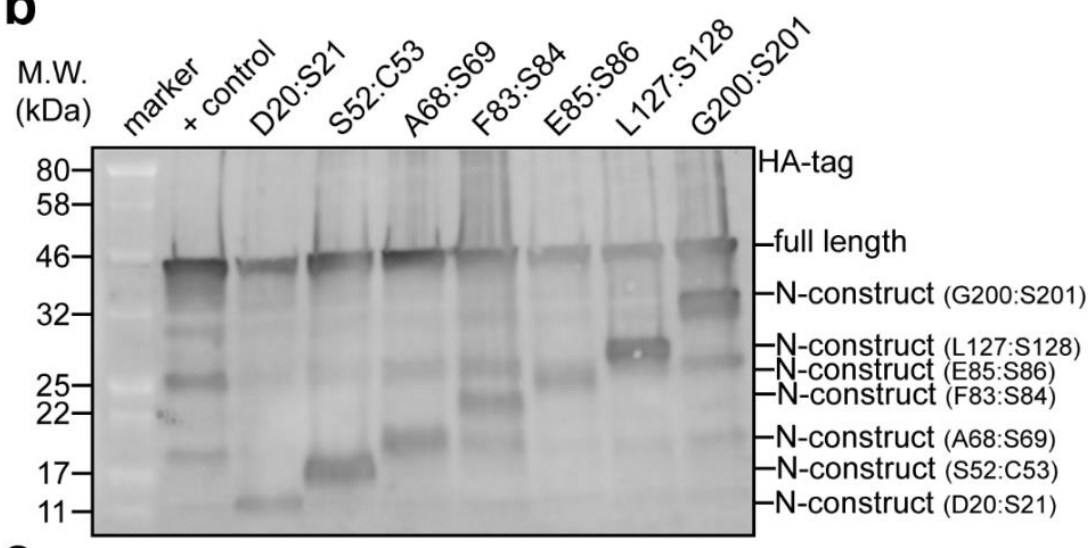

C
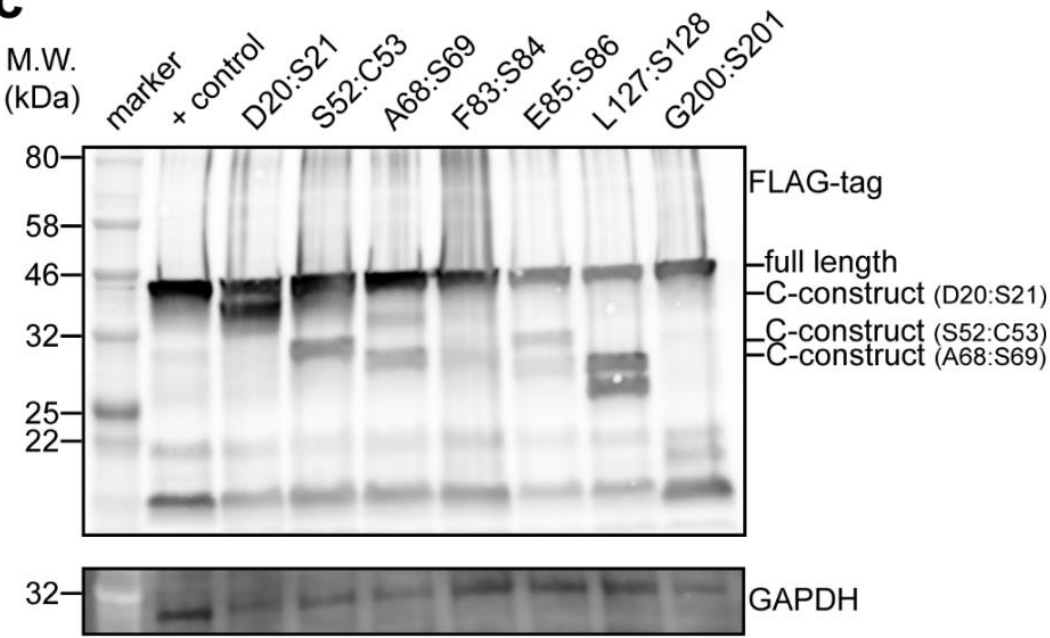

Figure S12. Hygromycin B phosphotransferase (HPT) is reconstituted by gp41-1-mediated protein splicing. (a) Schematics showing polypeptide fragments with predicted molecular weights. (b,c) Representative Western blot. The membrane was scanned with three different lasers to detect AlexaFluor 488-conjugated anti-rat secondary antibody directed against the rat anti-HA antibody (b), Cyanine5-conjugated anti-rabbit secondary antibody directed against the rabbit anti-FLAG antibody (c, upper image), and AlexaFluor 790-conjugated antimouse secondary antibody directed against the mouse anti-GAPDH antibody (c, lower image). GAPDH was used as loading control. 


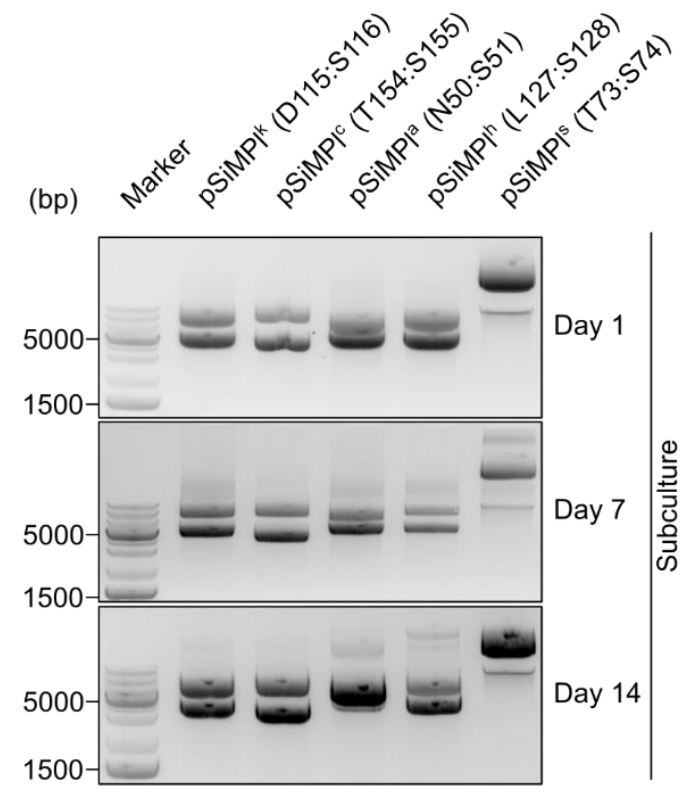

Figure S13. Stable maintenance of SiMPI plasmids in E. coli. Representative ethidium bromide-stained $1 \%$ agarose gel showing plasmid DNA isolated after 1, 7 and 14 day(s) of culturing from E. coli TOP10 cells transformed with the indicated SiMPl plasmid pairs grown continuously for 14 days in nutrient broth with the respective antibiotics. When using the $\mathrm{pSiMPl}^{\mathrm{s}}$ plasmid pair split at $\mathrm{T} 73: \mathrm{S} 74$, the two plasmids were not clearly distinguishable on the gel right from Day 1. Similar observation was also made when using the $\mathrm{pSiMPl}^{\mathrm{a}}$ plasmid pair split at N50:S51 at Day 14. In both these cases, the presence of both the plasmids was confirmed by Sanger sequencing. 
a

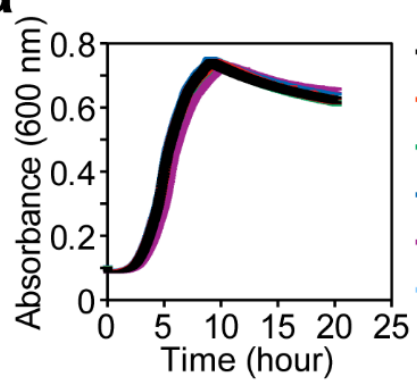

b

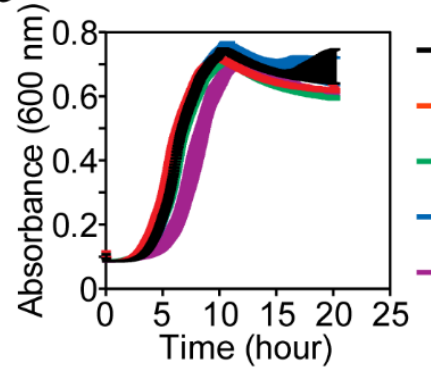

C
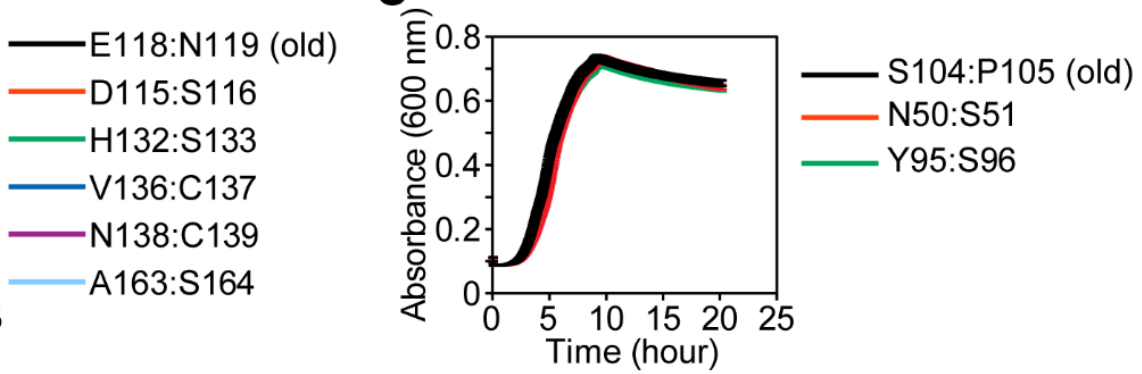

d

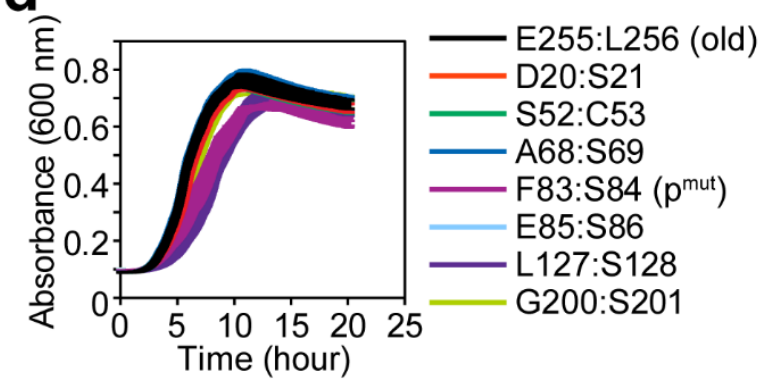

e

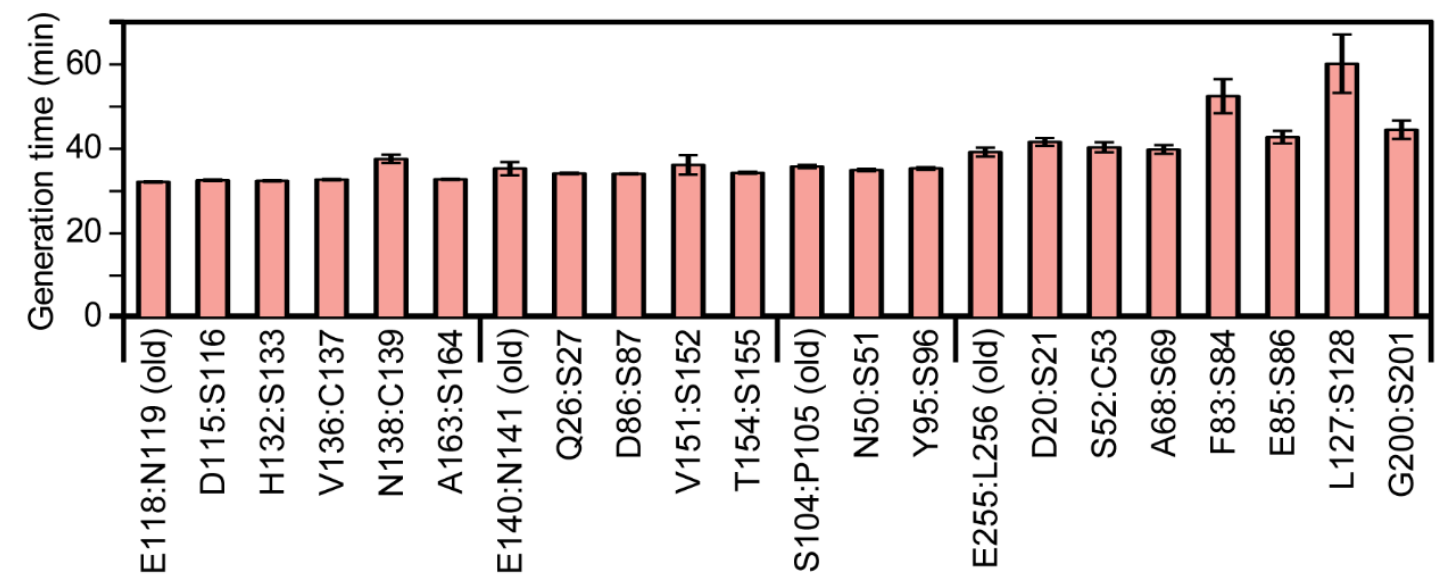

constructs

APT

CAT

TEM-1 $\beta$-L

HPT

Figure S14. Cells co-transformed with the pSiMPI plasmid pairs built splitting the enzymes at different sites grow similarly. (a-d) Growth curves of $E$. coli TOP10 cells transformed with pSiMPl plasmid pairs for use with kanamycin (a), chloramphenicol (b), ampicillin (c), and hygromycin (d) built splitting the enzymes at the indicated sites. $\mathrm{p}^{\text {mut }}$, mutated $\mathrm{P}_{\text {СAT }}$ promoter. For more information about $\mathrm{p}^{\text {mut }}$ see the legend to Figure S17. Old, previously identified splice site $^{2}$. Experiments were performed twice (biological replicates) with technical triplicates each time. Values represent mean \pm S.E.M. (e) Bar graph showing the generation time of bacteria grown in selective medium after transformation with the pSiMPl plasmid pair built splitting the enzymes at the indicated sites. The doubling time was calculated using data from (a-d) using the Growthcurver R package ${ }^{3}$. Values represent mean \pm S.E.M. 
a

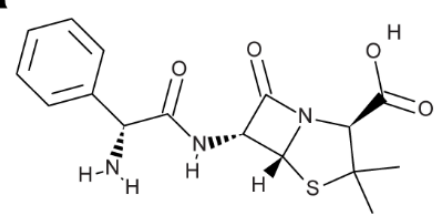

C

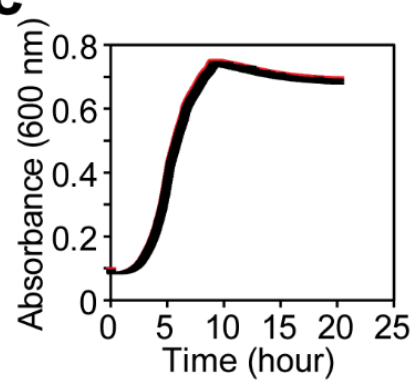

e

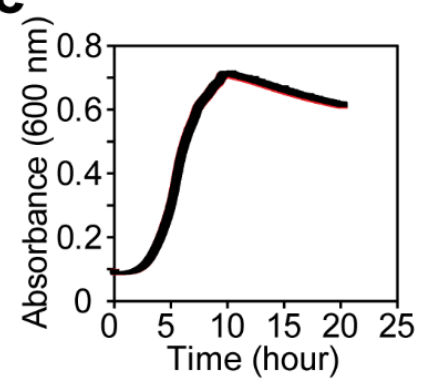

g

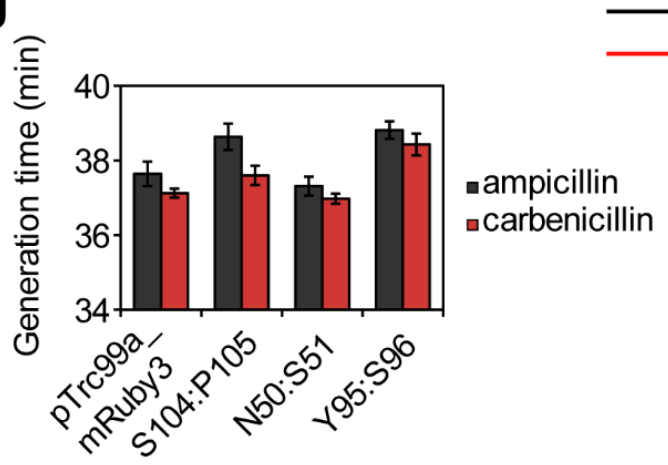

b

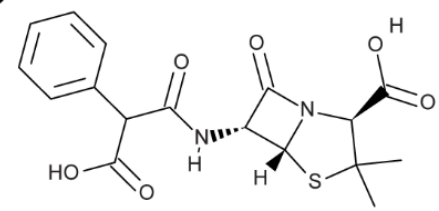

d

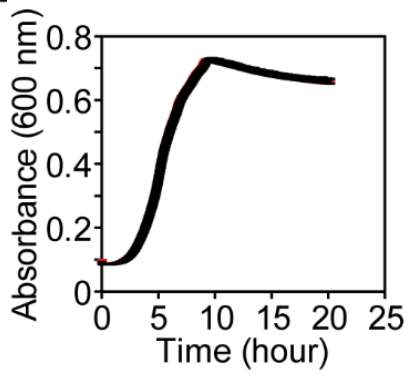

f

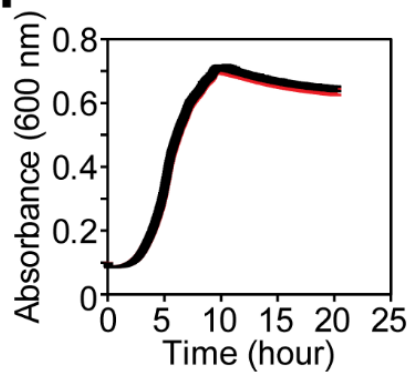

- ampicillin carbenicillin

Figure S15. Reconstituted TEM-1 $\beta$-lactamase expressed from $\mathrm{pSiMPl}^{\mathrm{a}}{ }_{-} \mathrm{N}$ and $\mathrm{pSiMPl}^{\mathrm{a}} \mathrm{C}$ allows for growth on carbenicillin. (a) Structure of ampicillin. (b) Structure of carbenicillin. (c-f) $\mathrm{OD}_{600}$ measurements of E. coli TOP10 cells transformed with either pTrc99a-mRuby3 (c), $\mathrm{pSiMPl}^{\mathrm{a}}$ (splice site: S104:P105; old; d), $\mathrm{pSiMPl}^{\mathrm{a}}$ (splice site: N50:S51; e) and $\mathrm{pSiMPl}^{\mathrm{a}}$ (splice site: $\left.\mathrm{Y} 95: \mathrm{S} 96 ; \mathbf{f}\right)$ grown in nutrient broth with the indicated antibiotic. Measurements were done in a plate reader for a total of 20 hours with each measurement taken every $2.4 \mathrm{~min}$. Experiments were performed twice (biological replicates) with technical triplicates each time. Values represent mean \pm S.E.M. (g) Generation time calculated using data from (c-f) with Growthcurver ${ }^{3}$. 
a

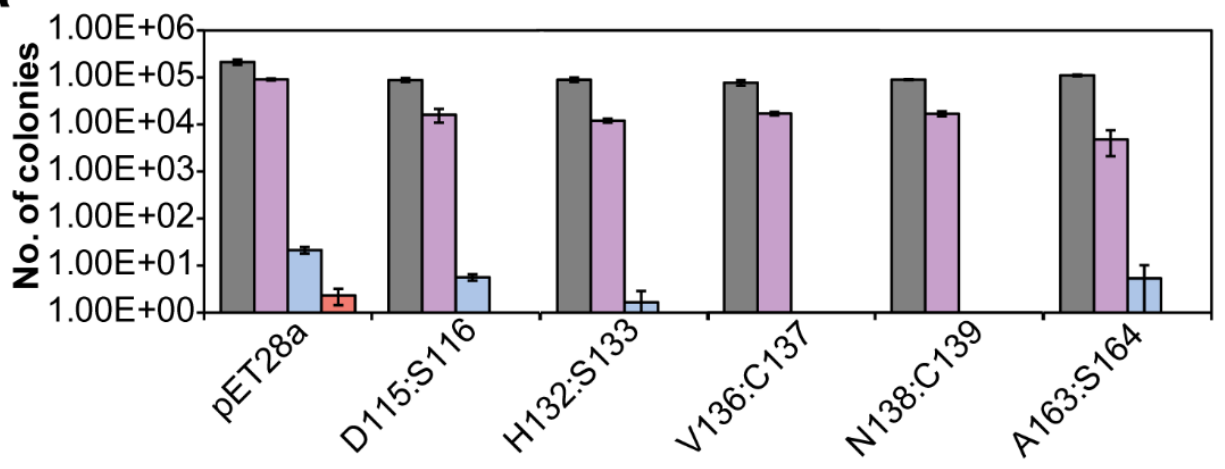

b

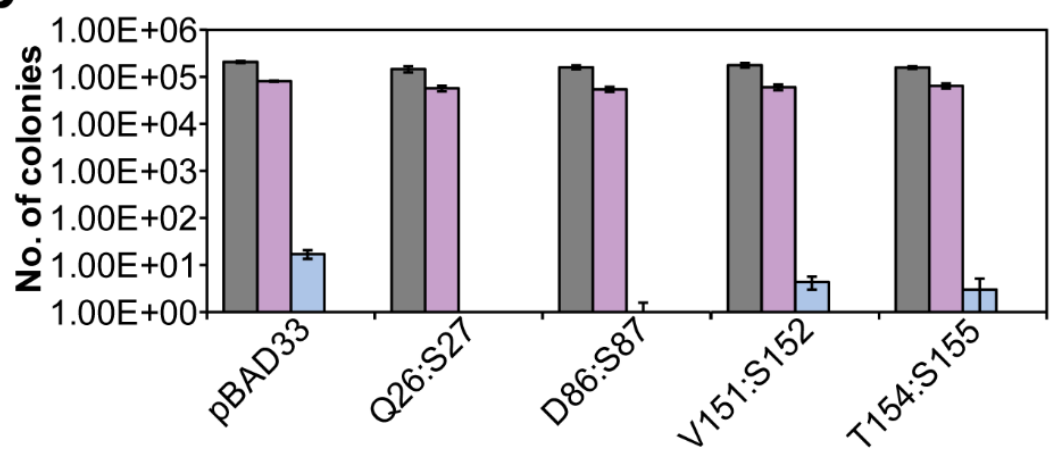

C

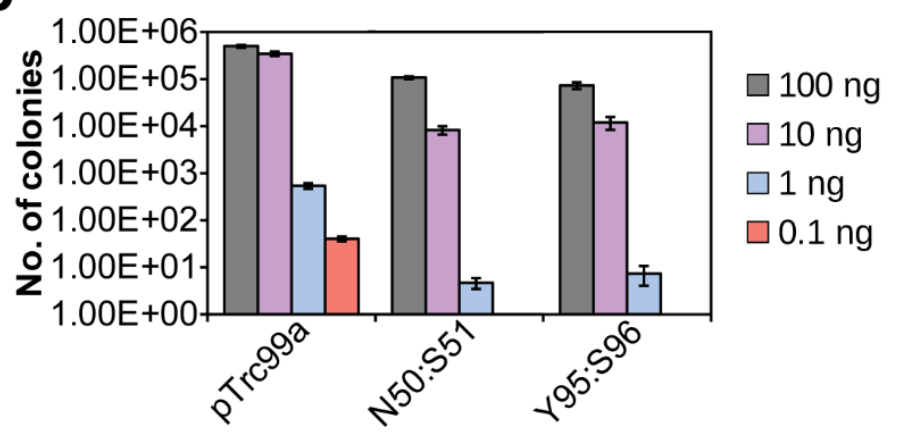

Figure S16. Transformation efficiency of pSiMPI plasmids decreases with decreasing amounts of DNA. (ac) Bar graph showing the number of colonies obtained after transformation of chemically competent $E$. coli TOP10 cells with the indicated plasmids at the indicated amount of total plasmid concentration. Values represent mean \pm S.E.M of three independent experiments. (a-b) color code as in (c). (a) kanamycin constructs, (b) chloramphenicol constructs and (c) ampicillin constructs. For the positive controls (pET28a, pBAD33 and pTrc99a) only one plasmid was transformed. In all other cases, the pSiMP1 plasmid pair was co-transformed. 
a
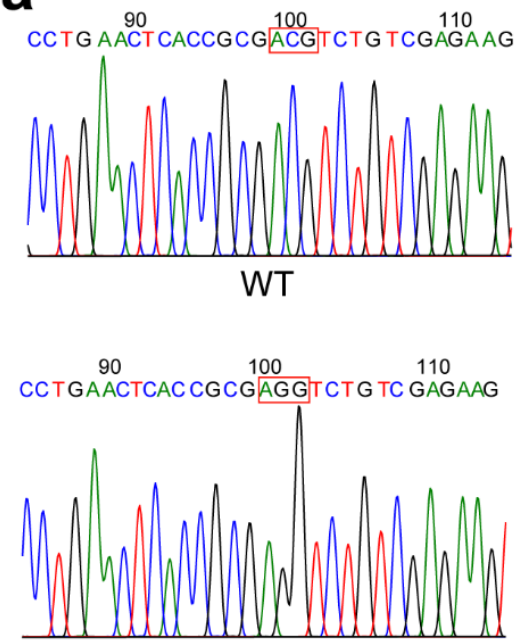

T9R

\section{C}

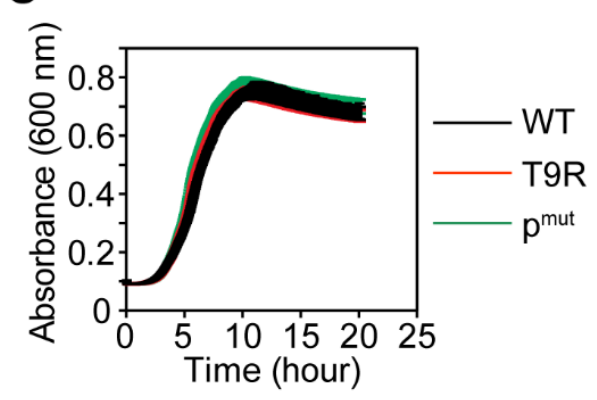

b

$\stackrel{40}{40} \stackrel{50}{60}$ AGTTATCGAGATTTCAGGAGCTAAGGAAGCT
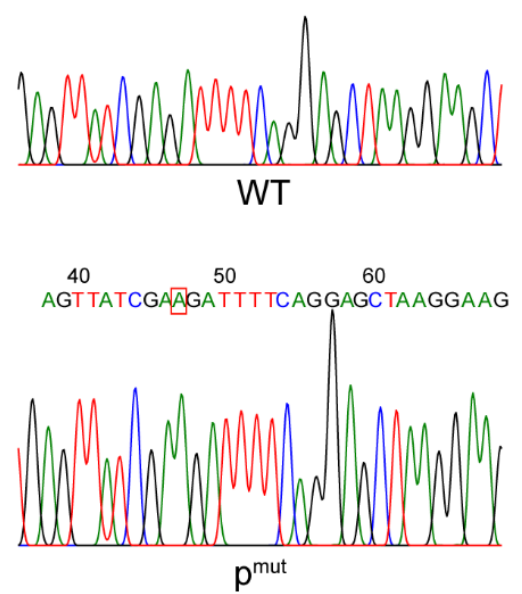

d

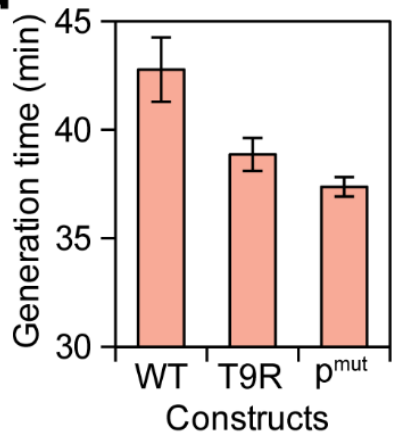

Figure S17. Some pSiMPl $^{\text {h_N }}$ constructs developed mutations. (a-b) Representative chromatograms showing (a) the transversion mutation ( $\mathrm{C}$ to $\mathrm{G}$ nucleotide change), which leads to the T9R mutation in the protein, and (b) the insertion mutation (insertion of nucleotide ' $\mathrm{A}$ ' between potential transcription start site and ribosome binding site) identified in a few $\mathrm{pSiMPl}^{\mathrm{h}}{ }_{-} \mathrm{N}$ clones with the splice site: E85:S86. (c) Growth curve of E. coli TOP10 cells co-transformed with $\mathrm{pSiMPl}^{\mathrm{h}}{ }_{-} \mathrm{C}$ and either $\mathrm{pSiMPl}^{\mathrm{h}}{ }_{-} \mathrm{N}$ (splice site: E85:S86, with no mutations), $\mathrm{pSiMPl}^{\mathrm{h}}{ }_{-} \mathrm{N}_{\text {with }}$ the transversion mutation $(\mathrm{T} 9 \mathrm{R})$ or $\mathrm{pSiMPl}^{\mathrm{h}}{ }_{-} \mathrm{N}$ with the insertion mutation $\left(\mathrm{p}^{\mathrm{mut}}\right)$ in the $\mathrm{P}_{\mathrm{CAT}}$ promoter. Note that this construct is different from the one that carries the same mutation in the $\mathrm{P}_{\text {CAT }}$ promoter shown in Figure S14, which is HPT split at F83:S84. OD 600 measurements were done in a plate reader for a total of 20 hours with each measurement taken every $2.4 \mathrm{~min}$. Experiments were performed twice (biological replicates) with technical triplicates each time. Values represent mean \pm S.E.M. (d) Generation time calculated using data from (c) with Growthcurver ${ }^{3}$. 


\section{a}

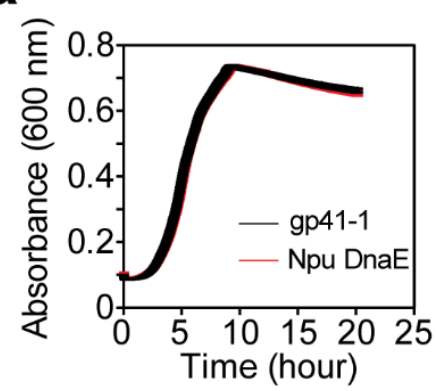

C

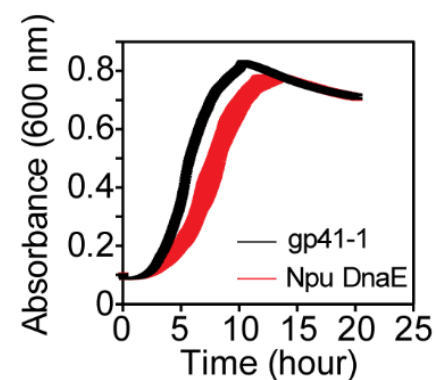

b

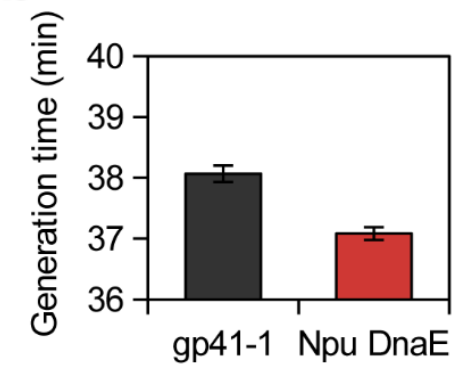

d

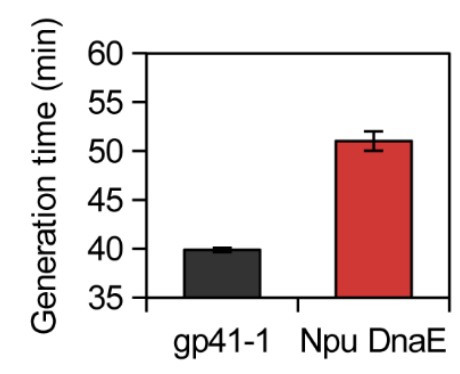

Figure S18. pSiMPI plasmids can be constructed with different split inteins. (a,c) $\mathrm{OD}_{600}$ measurements of $E$. coli TOP10 cells transformed with $\mathrm{pSiMPl}^{\mathrm{k}}$ (splice site: V136:C137; a) and $\mathrm{pSiMPl}^{\mathrm{h}}$ (splice site: $\mathrm{S} 52: \mathrm{C} 53 ; \mathbf{c}$ ) built using either the gp41-1 or Npu DnaE split intein. Measurements were done in a plate reader for a total of 20 hours with each measurement taken every $2.4 \mathrm{~min}$. Experiments were performed twice (biological replicates) with technical triplicates each time. Values represent mean \pm S.E.M. (b, d) Generation time calculated using data from (a) and (c) with Growthcurver ${ }^{3}$. 

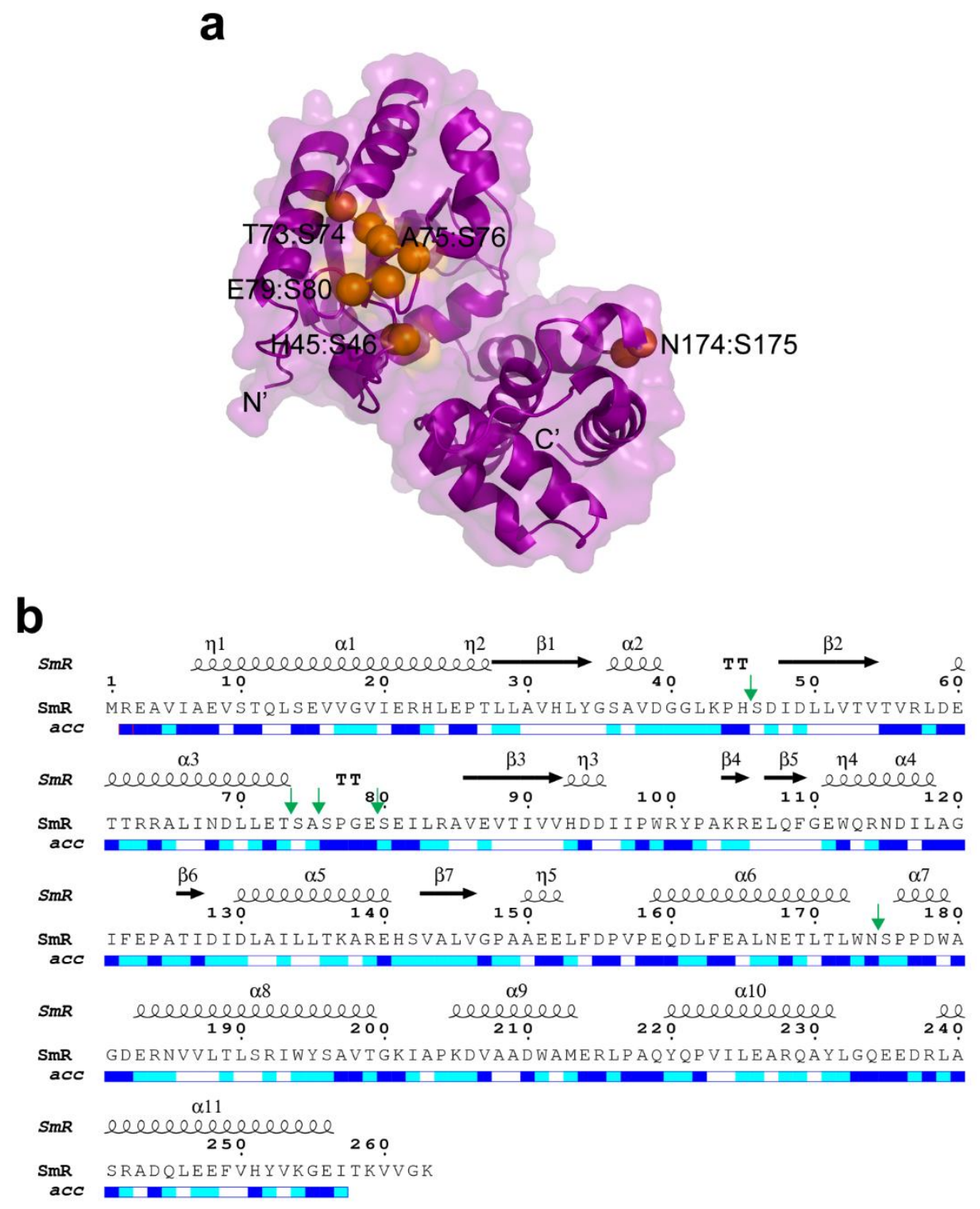

Figure S19. The identified splice sites in aminoglycoside adenyltransferase (SmR) are surface accessible. (a) Cartoon-surface representation of the structure of aminoglycoside adenyltransferase (SmR) modelled in SWISS-MODEL ${ }^{4}$ using PDB ID: $5 \mathrm{~g} 4 \mathrm{a}$ as template. The residues at the splice site are indicated by orange spheres. Structures were depicted in PyMOL (PyMOL Molecular Graphics System, v. 1.8.x, Schrödinger, LLC). (b) Relative accessibility (acc) of each residue in aminoglycoside adenyltransferase. Secondary structural elements are represented by black arrows and coils. The image was created using the ESPript 3.0 server $^{l}$. The color code is as follow: blue $=$ accessible, cyan $=$ partially accessible, white $=$ buried, red $=$ not predicted. Splice site are indicated by green arrows. 


\section{List of primers}

\begin{tabular}{|c|c|}
\hline Primer Name & Sequence $\left(5^{\prime}-3^{\prime}\right)$ \\
\hline Gib_SiMP1_BB1_FP & tgcttggatctgaaaacccaggttcagaccc \\
\hline Gib_SiMP1_BB1_RP & cattttagcttccttagctcctgaaaatctcgataactcaaaaaatacg \\
\hline Gib_SiMP1_BB2_FP & taactgtcagaccaagtttactcatatatactttagattgatttaaaacttcatttttaatttaaaagg \\
\hline Gib_SiMP1_BB2_RP & gttgtgggtcagaatatcgttagcgtaaaacaggtg \\
\hline RSP_Kan_FP & gagattttcaggagctaaggaagctaaaatgagccatattcaacgggaaacgtcttgc \\
\hline RSP_Kan_36_RP & tctgaacctgggttttcagatccaagcattgcccgacattatcgcgagccc \\
\hline RSP_Kan_116_RP & tctgaacctgggttttcagatccaagcaatcaggatattcttctaatacctggaatgctgttttccc \\
\hline RSP_Kan_133_RP & tctgaacctgggttttcagatccaagcaatgcaaccggcgcaggaacactg \\
\hline RSP_Kan_137_RP & tctgaacctgggttttcagatccaagcaaacaggaatcgaatgcaaccggcg \\
\hline RSP_Kan_139_RP & tctgaacctgggttttcagatccaagcaattacaaacaggaatcgaatgcaaccggc \\
\hline RSP_Kan_164_RP & tctgaacctgggttttcagatccaagcacgcatcaaccaaaccgttattcattcg \\
\hline RSP_Kan_RP & gtatatatgagtaaacttggtctgacagttagaaaaactcatcgagcatcaaatgaaactgc \\
\hline RSP_Kan_36_FP & ttacgctaacgatattctgacccacaactcaggtgcgacaatctatcgattgtatggg \\
\hline RSP_Kan_116_FP & ttacgctaacgatattctgacccacaactcaggtgaaaatattgttgatgcgctggc \\
\hline RSP_Kan_133_FP & ttacgctaacgatattctgacccacaactcgattcctgtttgtaattgtccttttaacagcgatcg \\
\hline RSP_Kan_137_FP & ttacgctaacgatattctgacccacaactgtaattgtccttttaacagcgatcgcgtatttcg \\
\hline RSP_Kan_139_FP & ttacgctaacgatattctgacccacaactgtccttttaacagcgatcgcgtatttcg \\
\hline RSP_Kan_164_FP & ttacgctaacgatattctgacccacaacagtgattttgatgacgagcgtaatggc \\
\hline RSP_Cam_FP & gagattttcaggagctaaggaagctaaaatggagaaaaaaatcactggatataccaccg \\
\hline RSP_Cam_14_RP & tctgaacctgggttttcagatccaagcatatatcaacggtggtatatccagtgattttttctcc \\
\hline RSP_Cam_27_RP & tctgaacctgggttttcagatccaagcactgaaatgcctcaaaatgttctttacgatgcc \\
\hline RSP_Cam_87_RP & tctgaacctgggttttcagatccaagcaatcccatatcaccagctcaccg \\
\hline RSP_Cam_152_RP & tctgaacctgggttttcagatccaagcacacccagggattggctgagacg \\
\hline RSP_Cam_155_RP & tctgaacctgggttttcagatccaagcaggtgaaactcacccagggattggc \\
\hline RSP_Cam_RP & gtatatatgagtaaacttggtctgacagttacgcccegccetgccactcat \\
\hline RSP_Cam_14_FP & ttacgctaacgatattctgacccacaactcccaatggcatcgtaaagaacattttgagg \\
\hline RSP_Cam_27_FP & ttacgctaacgatattctgacccacaactcagttgctcaatgtacctataaccagaccg \\
\hline RSP_Cam_87_FP & ttacgctaacgatattctgacccacaacagtgttcaccettgttacaccgttttcc \\
\hline RSP_Cam_152_FP & ttacgctaacgatattctgacccacaacagtttcaccagttttgatttaaacgtggcc \\
\hline RSP_Cam_155_FP & ttacgctaacgatattctgacccacaacagttttgatttaaacgtggccaatatggacaac \\
\hline
\end{tabular}




\begin{tabular}{|c|c|}
\hline RSP_Amp_FP & gagattttcaggagctaaggaagctaaaatgagtattcaacatttccgtgtcgecettattccc \\
\hline RSP_Amp_51_RP & tctgaacctgggttttcagatccaagcagttgagatccagttcgatgtaacccactcgtg \\
\hline RSP_Amp_96_RP & tctgaacctgggttttcagatccaagcaatagtgtatgcggcgaccgagttgctc \\
\hline RSP_Amp_221_RP & tctgaacctgggttttcagatccaagcagcgcagaagtggtcctgcaactttatcc \\
\hline RSP_Amp_264_RP & tctgaacctgggttttcagatccaagcacccegtcgtgtagataactacgatacgggag \\
\hline RSP_Amp_RP & gtatatatgagtaaacttggtctgacagttaccaatgcttaatcagtgaggcacctatctcagcg \\
\hline RSP_Amp_51_FP & ttacgctaacgatattctgacccacaacagcggtaagatccttgagagttttcgecc \\
\hline RSP_Amp_96_FP & ttacgctaacgatattctgacccacaactctcagaatgacttggttgagtactcaccagtcacag \\
\hline RSP_Amp_221_FP & ttacgctaacgatattctgacccacaactcggecettccggctggctggtttattgc \\
\hline RSP_Amp_264_FP & $\begin{array}{l}\text { ttacgctaacgatattctgacccacaacagtcaggcaactatggatgaacgaaatagacagat } \\
\text { cg }\end{array}$ \\
\hline RSP_Hyg_FP & gagattttcaggagctaaggaagctaaaatgaaaaagcctgaactcaccgcgacgtctg \\
\hline RSP_Hyg_21_RP & $\begin{array}{l}\text { tctgaacctgggttttcagatccaagcagtcgaacttttcgatcagaaacttctcgacagacgtc } \\
\mathrm{g}\end{array}$ \\
\hline RSP_Hyg_53_RP & tctgaacctgggttttcagatccaagcagctatttacccgcaggacatatccacgccetcc \\
\hline RSP_Hyg_69_RP & $\begin{array}{l}\text { tctgaacctgggttttcagatccaagcatgcaaagtgccgataaacataacgatcttgtagaa } \\
\text { accatcgg }\end{array}$ \\
\hline RSP_Hyg_84_RP & tctgaacctgggtttcagatccaagcagaattccccaatgtcaagcacttccggaatcgg \\
\hline RSP_Hyg_86_RP & tctgaacctgggttttcagatccaagcactcgctgaattccccaatgtcaagcacttccg \\
\hline RSP_Hyg_128_RP & tctgaacctgggttttcagatccaagcaaagatcggccgcagcgatcgcatc \\
\hline RSP_Hyg_201_RP & tctgaacctgggttttcagatccaagcagccgaaatccgcgtgcacgaggtg \\
\hline RSP_Hyg_RP & gtatatatgagtaaacttggtctgacagttattcctttgccctcggacgagtgctgg \\
\hline RSP_Hyg_21_FP & ttacgctaacgatattctgacccacaacagcgtctccgacctgatgcagctctc \\
\hline RSP_Hyg_53_FP & $\begin{array}{l}\text { ttacgctaacgatattctgacccacaactgcgecgatggtttctacaaagatcgttatgtttatcg } \\
\text { gcac }\end{array}$ \\
\hline RSP_Hyg_69_FP & ttacgctaacgatattctgacccacaactcggccgcgetcccgattcc \\
\hline RSP_Hyg_84_FP & ttacgctaacgatattctgacccacaacagcgagagcctgacctattgcatctccc \\
\hline RSP_Hyg_86_FP & ttacgctaacgatattctgacccacaacagcctgacctattgcatctccegccg \\
\hline RSP_Hyg_128_FP & ttacgctaacgatattctgacccacaacagccagacgagcgggttcggc \\
\hline RSP_Hyg_201_FP & ttacgctaacgatattctgacccacaactccaacaatgtcctgacggacaatggccg \\
\hline pET_HA_Kan_FP & $\begin{array}{l}\text { aaacagtaatacaaggggtgttatgtacccatacgatgttccagattacgctggcagccatatt } \\
\text { caacgggaaacgtcttgc }\end{array}$ \\
\hline pET_Kan_RP & tatgtatccgctcatgaattaattcttagaaaaactcatcgagcatcaaatgaaactgc \\
\hline
\end{tabular}




\begin{tabular}{|c|c|}
\hline pET_no Kan_FP & taagaattaattcatgagcggatacatatttgaatgtatttagaaaaataaac \\
\hline pET_no Kan_RP & cataacacccttgtattactgtttatgtaagcagacag \\
\hline pBAD_HA_Cam_FP & $\begin{array}{l}\text { attttcaggagctaaggaagctaaaatgtacccatacgatgttccagattacgctggcgagaa } \\
\text { aaaaatcactggatataccaccgttgatatatccc }\end{array}$ \\
\hline $\begin{array}{l}\text { pBAD_FLAG_Cam_ } \\
\text { RP }\end{array}$ & $\begin{array}{l}\text { ggcaccaataactgecttaaaaaaattacttgtcgtcatcgtctttgtagtccgeccegccetgc } \\
\text { cactcatcgcag }\end{array}$ \\
\hline pBAD_no Cam_FP & taatttttttaaggcagttattggtgccettaaacgcctggtgctacg \\
\hline pTrc_Amp_FP & gcttcaataatattgaaaaaggaagagtatgagtattcaacatttccgtgtcgeccttattccc \\
\hline $\begin{array}{l}\text { pTrc_FLAG_Amp_R } \\
\text { P }\end{array}$ & $\begin{array}{l}\text { gtatatatgagtaaacttggtctgacagttacttgtcgtcatcgtctttgtagtcccaatgcttaatc } \\
\text { agtgaggcacctatc }\end{array}$ \\
\hline pTrc_no Amp_RP & catactcttccttttcaatattattgaagcatttatcaggg \\
\hline pBAD_HA_Hyg_FP & $\begin{array}{l}\text { attttcaggagctaaggaagctaaaatgtacccatacgatgttccagattacgctggcggtaaa } \\
\text { aagcctgaactcaccgcgac }\end{array}$ \\
\hline $\begin{array}{l}\text { pBAD_FLAG_Hyg_ } \\
\text { RP }\end{array}$ & $\begin{array}{l}\text { ggcaccaataactgccttaaaaaaattacttgtcgtcatcgtctttgtagtcttcctttgccctcgg } \\
\text { acgagtgctg }\end{array}$ \\
\hline pBAD_HA_Kan_FP & $\begin{array}{l}\text { attttcaggagctaaggaagctaaaatgtacccatacgatgttccagattacgctggcagccat } \\
\text { attcaacgggaaacgtcttgc }\end{array}$ \\
\hline $\begin{array}{l}\text { pTrc_FLAG_Cam_R } \\
\text { P }\end{array}$ & $\begin{array}{l}\text { gtatatatgagtaaacttggtctgacagttacttgtcgtcatcgtctttgtagtccgcccegccet } \\
\text { gccactcatcgcag }\end{array}$ \\
\hline pBAD_Amp_FP & atttcaggagctaaggaagctaaaatgagtattcaacatttccgtgtcgcccttattccc \\
\hline pTrc_FLAG_Hyg_RP & $\begin{array}{l}\text { gtatatatgagtaaacttggtctgacagttacttgtcgtcatcgtctttgtagtcttcctttgccctc } \\
\text { ggacgagtgctg }\end{array}$ \\
\hline npu_Kan_137_FP & $\begin{array}{l}\text { gaagaacggcttcatcgcctccaactgtaattgtcctttaacagcgatcgcgtatttcgtctcg } \\
\text { c }\end{array}$ \\
\hline npu_Kan_137_RP & ggatctcggtttcgtaggacaagcaaacaggaatcgaatgcaaccggcgcaggaac \\
\hline npu_Kan_139_FP & gaagaacggcttcatcgcctccaactgtccttttaacagcgatcgcgtatttcgtctcgc \\
\hline npu_Kan_139_RP & ggatctcggtttcgtaggacaagcaattacaaacaggaatcgaatgcaaccggcgcagg \\
\hline npu_Hyg_53_FP & $\begin{array}{l}\text { gaagaacggcttcatcgcctccaactgcgccgatggtttctacaaagatcgttatgtttatcgg } \\
\text { cac }\end{array}$ \\
\hline npu_Hyg_53_RP & ggatctcggtttcgtaggacaagcagctatttacccgcaggacatatccacgccctcc \\
\hline npu_RSP_N_FP & tgcttgtcctacgaaaccgagatcctgaccgtgg \\
\hline npu_RSP_N_RP & ggcaccaataactgccttaaaaaaattattagttgggcaggttgtccacgcgcatcaggtcc \\
\hline npu_RSP_C_FP & gcttcaataatattgaaaaaggaagagtatgatcaagatcgccacccgcaagtacctgggc \\
\hline
\end{tabular}


\begin{tabular}{|l|l|}
\hline npu_RSP_C_RP & gttggaggcgatgaagccgttcttcagggcg
\end{tabular}

\section{References}

[1] Robert, X., and Gouet, P. (2014) Deciphering key features in protein structures with the new ENDscript server, Nucleic Acids Res 42, W320-324.

[2] Palanisamy, N., Degen, A., Morath, A., Ballestin Ballestin, J., Juraske, C., Ozturk, M. A., Sprenger, G. A., Youn, J. W., Schamel, W. W., and Di Ventura, B. (2019) Split intein-mediated selection of cells containing two plasmids using a single antibiotic, Nat Commun 10, 4967.

[3] Sprouffske, K., and Wagner, A. (2016) Growthcurver: an R package for obtaining interpretable metrics from microbial growth curves, BMC Bioinformatics 17, 172.

[4] Waterhouse, A., Bertoni, M., Bienert, S., Studer, G., Tauriello, G., Gumienny, R., Heer, F. T., de Beer, T. A. P., Rempfer, C., Bordoli, L., Lepore, R., and Schwede, T. (2018) SWISS-MODEL: homology modelling of protein structures and complexes, Nucleic Acids Res 46, W296-W303. 